Relations industrielles

Industrial Relations

\title{
À l'enseigne du droit social belge, Troisième édition revue et augmentée, in Revue de l'Université de Bruxelles, 1978, Bruxelles, Éditions de l’Université de Bruxelles, 1982, 392 pp.
}

\section{Pierre Verge}

Volume 38, numéro 2, 1983

URI : https://id.erudit.org/iderudit/029367ar

DOI : https://doi.org/10.7202/029367ar

Aller au sommaire du numéro

\section{Éditeur(s)}

Département des relations industrielles de l'Université Laval

\section{ISSN}

0034-379X (imprimé)

1703-8138 (numérique)

Découvrir la revue

Citer ce compte rendu

Verge, P. (1983). Compte rendu de [À l'enseigne du droit social belge, Troisième édition revue et augmentée, in Revue de l'Université de Bruxelles, 1978, Bruxelles, Éditions de l'Université de Bruxelles, 1982, 392 pp.] Relations industrielles / Industrial Relations, 38(2), 445-446.

https://doi.org/10.7202/029367ar

Tous droits réservés (C) Département des relations industrielles de l'Université Laval, 1983
Ce document est protégé par la loi sur le droit d'auteur. L'utilisation des services d'Érudit (y compris la reproduction) est assujettie à sa politique d'utilisation que vous pouvez consulter en ligne.

https://apropos.erudit.org/fr/usagers/politique-dutilisation/ 
susceptibles de donner ouverture à des mesures patronales. Les auteurs tentent de plus une distinction entre les mesures disciplinaires et non disciplinaires ainsi qu'une présentation détaillée des diverses mesures comprises sous chaque rubrique.

L'analyse est de qualité et elle traduit une conception qui tend à mettre en évidence l'émergence d'un droit de gestion des ressources humaines.

Cette façon de voir est intéressante et il est à espérer que d'autres auteurs tenteront de préciser davantage le contenu de ce droit. Il est par ailleurs souhaitable qu'une telle démarche s'effectue non pas à l'ombre du droit civil (contrat individuel de travail), mais qu'elle s'en affranchisse.

La seconde partie est d'abord consacrée à l'étude des diverses règles relatives aux formes d'imposition des mesures correctives ou à certaines exigences préalables dont le respect doit être assuré pour justifier lesdites mesures. Suit une présentation des divers manquements du salarié. On peut y déceler, à regret, une volonté manifeste des auteurs de rattacher à chacun de ces manquements une obligation correspondante du contrat individuel de travail. La présentation de ces divers manquements obéit à la distinction entre les mesures disciplinaires et non disciplinaires.

Tout comme dans la première partie on peut reconnaître ici un texte bien structuré et de belle rédaction.

Praticiens, chercheurs et étudiants trouveront dans cet ouvrage une synthèse intéressante sur les tendances de la jurisprudence québécoise arbitrale en matière de contrôle du bien-fondé ou non de l'imposition des mesures disciplinaires et non disciplinaires.

Rodrigue BLOUIN

Université Laval

À l'enseigne du droit social belge, Troisième édition revue et augmentée, in Revue de
l'Université de Bruxelles, 1978, Bruxelles, Éditions de l'Université de Bruxelles, 1982, $392 \mathrm{pp}$.

Cet ouvrage consiste dans une juxtaposition de tableaux du droit belge du travail et de la sécurité sociale rédigés par plusieurs collaborateurs, sous la direction de M. Pierre Van der Vorst, de la Faculté de droit de l'Université Libre de Bruxelles. Bien qu'il ne s'agisse point là d'une présentation intégrée et systématique de ces systèmes, l'ampleur des sujets retenus, la teneur plutôt fondamentale des propos, de même que l'abondance de la documentation à laquelle l'on se réfère, ont néanmoins pour résultat de sensibiliser fort utilement le lecteur étranger aux grands aspects de ce droit belge contemporain et de son évolution.

Le directeur de l'entreprise, sous le titre "Clefs du droit social belge», ouvre toutes grandes les portes en formulant les traits fondamentaux du droit social, qu'il envisage essentiellement dans son contexte national, mais aussi à la lumière des événements et des auteurs qui l'ont façonné en France. Ce droit social, pose-t-on, est relativement homogène sous son «apparence fragmentée». S'il connaît des emprunts à d'autres secteurs du droit, «... ce n'est pas pour s'identifier avec ces branches définies du droit mais parce qu'elles sont pour lui des moyens techniques d'atteindre l'objectif social qu'il se propose ... jamais il n'hésitera à déformer le visage des disciplines dont il se sert et lorsque cellesci seront un carcan pour ses finalités, il ne manquera pas de les ignorer par un bouleversement complet de leur économie ou par une formulation originale» (p. 22). L'exposé de la gestation historique du droit belge, l'émergence d'un droit notamment «protecteur, organisateur, réformiste, progressif, stratifié» débouche ensuite sur une vérification plus détaillée de la prise de position précédente, sur un état des ambiguïtés du droit social belge. Non seulement, en particulier, son ambivalence idéologique entre diverses formes d'interventionnisme et «divers types de libéralisme», ses hésitations devant divers concepts juridiques (par exemple, à l'occa- 
sion de l'analyse de la nature de la grève et de ses effets), mais surtout peut-être l'importance du "non droit», en particulier dans le cours final de la négociation collective: «le droit positif peut y être délibérément baillonné au profit de solutions circonstancielles» (p. 79). Le texte suivant, de M. Magrez, «Les principes généraux du droit social ou l'ordre cohérent», poursuit la réflexion à ce niveau. Fait révélateur de l'importance du rôle de la négociation interprofessionnelle et nationale dans la réalisation de réformes sociales et de son influence subséquente parfois sur le législateur lui-même, suit immédiatement un texte de madame Magrez-Song intitulé: «Le rôle des conventions collectives ou l'élaboration parallèle».

Des études plus spécifiques peut-être, mais non moins importantes, sont ensuite consacrées à l'«avenir de la sécurité sociale» (J. Gillain), à celui du droit du travail (M. Taquet et $C$. Wantiez) - on y déplore certaines carences du régime du licenciement, dont l'absence étonnante d'une obligation de motivation - à l'«expérience des amendes administratives et aux aspects fondamentaux du droit pénal social» (P. Gosseries), au rôle du droit et de la jurisprudence communautaires (E. Vogel-Polsky), à l'apport des statistiques en droit social (A. Delpérée). On verra aussi sous le titre «Les juridictions du travail» (U. Deprez), comment la Belgique a réalisé, en 1967, l'unification des diverses juridictions sociales en intégrant les instances du travail au pouvoir judiciaire ordinaire, les magistrats de carrière siègeant avec d'autres juges et conseillers sociaux nommés pour une période déterminée sur présentation des organisations représentatives des travailleurs salariés, des employeurs et des travailleurs indépendants. Leur compétence, en matière de droit du travail, observons le en passant (p. 248), ne s'étend pas au règlement des conflits collectifs du travail, mais se limite aux conflits relatifs à des droits subjectifs, issus du contrat de travail.

Les trois dernières contributions sont, chacune, d'un apport bien particulier. Il s'agit, en premier lieu, de cet imposant «Essai de bibliographie du droit social belge" (M. Sojcher - Rousselle et G. Desolre): une présentation thématique des principaux ouvrages et documents, tant de langue néerlandaise que française, par autant de sommaires qui auront l'heur, en particulier, de guider le comparatiste. Finalement, deux regards d'ensemble sur le droit social belge. L'un d'outrefrontière, celui du professeur Gérard LyonCaen pour qui, notamment, la Belgique demeure avant tout la "terre du paritarisme», de la négociation collective institutionnalisée généralisée et hiérarchisée. L'autre, du professeur Lucien François, de l'Université de Liège, dont la proposition à l'effet d'en venir à un "système d'élections, pour régler sans arbitraire la participation des syndicats au pouvoir" véhicule d'importantes réserves quant à la façon pour l'État d'investir ces associations privées que sont les principales centrales syndicales de son pays d'une mission de négociation à ce point liée à l'intérêt public. Bien que dites "représentatives», ces influents organismes, souligne-t-on, se sont, en fait qu'agrées. "Tous ces privilèges restreignent la liberté syndicale, altèrent le caractère de notre système politique, et transforment progressivement les syndicats en institutions influentes, mais liées, d'un État qui devient corporatif» (p. 390). Note discordante, sans doute sévère mais salutaire - on ne peut en juger - par rapport à un ensemble où l'identité du droit social paraît déjà marquée.

Pierre VERGE

Université Laval

The Arbitration Guide: a Case-handing Manual of Procedures and Practices in Dispute Resolutions, par Raymond L. Britton, Englewood Cliffs, new Jersey, Prentice Hall, Inc., 1982, 298 pp. ISBN 0-13-043984-3

Ce document se veut un "vade mecum» s'adressant tout particulièrement aux praticiens exerçant dans le domaine de l'arbitrage. Son objectif est de permettre un accès rapide à des informations générales ou fondamenta- 